\title{
Panic prescribing has become omnipresent during the COVID-19 pandemic
}

\author{
Arthur L. Caplan' and Ross Upshur r,3,4 \\ 'Division of Medical Ethics, New York University Grossman School of Medicine, New York, New York, USA. ${ }^{2}$ Department of Family and Community Medicine and ${ }^{3}$ Dalla Lana School of Public Health, University \\ of Toronto, Toronto, Ontario, USA. ${ }^{4}$ Lunenfeld-Tanenbaum Research Institute, Sinai Health, Toronto, Ontario, Canada.
}

$T_{h}$ he President of the United States has repeatedly touted hydroxychlororquine as a likely cure for COVID-19 and urged Americans to try it, stating at one of his media briefings, "What do you have to lose? What do you have to lose? Take it" (1). A few others around the world have chimed in to promote one drug or another, this drug in combination with others, or their own favorite untested nostrums. This has led to drug hoarding, the inability of patients who actually need and benefit from certain drugs to access them, and serious side effects and even deaths from self-medication.

\section{Unregistered, untried therapeutics}

Little noted has been the fact that the world has faced this sort of panic prescribing and recommendation to abandon all efforts at organized study before. Six years ago, on August 12, 2014, the Ebola outbreak led the World Health Organization (WHO) to convene a meeting to decide whether to proceed with evaluation of unregistered, untried therapeutics.

\section{Press release}

"In the particular circumstances of this outbreak, and provided certain conditions are met, the panel reached consensus that it is ethical to offer unproven interventions with as yet unknown efficacy and adverse effects, as potential treatment or prevention.

Ethical criteria must guide the provision of such interventions. These include transparency about all aspects of care, informed consent, freedom of choice, confidentiality, respect for the person, preservation of dignity, and involvement of the community.
In order to understand the safety and efficacy of these interventions, the group advised that, if and when they are used to treat patients, there is a moral obligation to collect and share all data generated, including from treatments provided for 'compassionate use' (access to an unapproved drug outside of a clinical trial)" (2).

As it was unclear at the time whether any benefit would come from these interventions, a mechanism called monitored emergency use of unregistered and investigational interventions (MEURI) was created as a bridge to clinical trials in order to curtail unconstrained use of medications.

By August 12, 2019, the Data and Safety Monitoring Board recommended early termination of a Democratic Republic of the Congo (DRC) randomized control trial; by October 18, 2019, a safe vaccine had been identified.

\section{Accelerated implementation} and evaluation of interventions After 5 years, the world went from a situation where Ebola infection was regarded as a dreaded, nearly universally fatal disease that could neither be treated nor prevented to a disease with a registered vaccine and two monoclonal antibodies with significantly positive treatment effects (3, 4). What is worth noting is the accelerated implementation and evaluation of these interventions - the global coordination and funding to go from a situation of therapeutic nihilism, panic, and despair to one of optimism and hope.

The most remarkable aspect of this narrative is that the research was carried out in some of the most rudimentary and under-resourced health systems in the world. Clinical trials were initiated in

Conflict of interest: The authors have declared that no conflict of interest exists.

Copyright: (c) 2020, American Society for Clinical Investigation.

Reference information: J Clin Invest. 2020;130(6):2752-2753. https://doi.org/10.1172/JC1139562.

West Africa from 2014 to 2016. However, despite mathematical models predicting large numbers of cases, the outbreak was ebbing when the clinical trials started in December 2014. Even then, it was possible to get trials designed and approved in less than four months. The final clinical trial in the DRC took place under exceptionally difficult circumstances, including civil conflict and political instability. Moreover, the research was conducted according to good clinical practice standards, was approved by local and international ethics committees, and met agreed-upon standards for the protection of human subjects. In this case, the science and ethics worked well together, and a noteworthy result occurred (5).

It would be foolish to argue that the path for studying treatments for Ebola was entirely smooth. There were principled disagreements on the nature of study designs, the inclusion of placebo controls, the nature of community engagement, and the adequacy of informed consent, particularly for the collection, storage, and transport of data and tissues. However, the debates led to consensus $(6,7)$.

These lessons are clearly applicable to the SARS-CoV-2 pandemic. It is possible to develop an effective clinical research response that can generate answers to priority questions in a rapid fashion. This is the intention behind the Solidarity trial, a multinational adaptive randomized platform design (8). The virtue of this design is the capacity to discard ineffective treatments and add promising options for evaluation in the same core protocol. Efforts should be devoted to participating in well-founded trials and avoiding small, underpowered trials, as they are noninformative and squander resources.

Clinical trials in infectious disease emergencies can be conducted without derogating human subjects' protection and watering down research ethics stan- 
dards. This is made clear in the WHO's guidance statement on research ethics in the response to the pandemic (9). Research ethics boards should be gearing up and ready to rapidly evaluate studies. There may be a role for MEURI in COVID-19, but unconstrained, unevaluated use of therapeutics under the guise of compassionate use or panicked rhetoric about right-to-try must be aggressively discouraged in order for scientists to learn what regimens or vaccines actually work.

The fact is, without organization, systematic investigations for the hundreds of agents purported to prevent seroconversion after infection, to treat those infected, or to find prophylactic vaccines will cause the world to spend scarce resources, spinning rapidly to the next wonder-agent proposed by those with little understanding of drug development or those who stand to gain financially. We have, at the ready, study designs, ethical protections, and methodological flexibility adequate to proceed quickly - but not blindly - to find answers. The way forward is not medicine by anecdote, panic prescribing, hoarding, or testimonials. The way to find safe and effective interventions is to use adaptive platforms of adequate power alongside compassionate use of novel agents in a manner that permits the accumulation of knowledge. Hope drives research to find answers - it's not a substitute for solutions.

\section{Acknowledgments}

ALC wishes to acknowledge the feedback of members of the NYU School of Medicine Working Group on Compassionate Use and Preapproval Access.

Address correspondence to: Arthur L. Caplan, 227 East 30th Street 7th Floor Room 722, New York, New York 10016, USA. Email: arthur.caplan@nyulangone.org.

1. Lynch HF, Bateman-House A, Caplan AL. 'Panic Prescribing' Untested Coronavirus Treatments: A Danger To Patients Today and Tomorrow. Health Affairs Blog. March 31, 2020. https://www.healthaffairs.org/do/10.1377/ hblog20200330.265604/full. Accessed April 27, 2020.

2. WHO. Ethical considerations for use of unregistered interventions for Ebola virus disease (EVD): Summary of the Panel Discussion. https://www. who.int/mediacentre/news/statements/2014/ ebola-ethical-review-summary/en. Updated
August 12, 2014. Accessed April 27, 2020.

3. Henao-Restrepo AM, et al. Efficacy and effectiveness of an rVSV-vectored vaccine in preventing Ebola virus disease: final results from the Guinea ring vaccination, open-label, clusterrandomised trial (Ebola Ça Suffit!). Lancet. 2017;389(10068):505-518.

4. Mulangu S, et al. A randomized, controlled trial of Ebola virus disease therapeutics. N Engl J Med. 2019;381(24):2293-2303.

5. Schopper D, et al. Research ethics governance in times of Ebola. Public Health Ethics. 2017;10(1):49-61.

6. Eccleston-Turner, et al. Inter-institutional relationships in global health: regulating coordination and ensuring accountability. Global Health Governance. 2018;12(2):83-97.

7. WHO. Ethical issues related to study design for trials on therapeutics for Ebola Virus Disease: WHO Ethics Working Group meeting 20-21 October, Summary of Discussion. Geneva, Switzerland: WHO; 2014.

8. [No authors listed]. "Solidarity" clinical trial for COVID-19 treatments. World Health Organization. https://www.who.int/emergencies/ diseases/novel-coronavirus-2019/globalresearch-on-novel-coronavirus-2019-ncov/ solidarity-clinical-trial-for-covid-19-treatments. Accessed May 5, 2020.

9. WHO. Ethical standards for research during public health emergencies: Distilling existing guidance to support COVID-19 R\&D. Geneva, Switzerland: WHO; 2020. 